\title{
Rapid Interchangeable Hydrogen, Hydride and Proton Species at the Interface of Transition Metal Atom on Oxide Surface
}

\author{
Simson Wu, ${ }^{1}$ Kai-Yu Tseng, ${ }^{2}$ Ryuichi Kato, ${ }^{3}$ Tai-Sing Wu, ${ }^{4,5}$ Alexander Large, ${ }^{6}$ Yung-Kang Peng, ${ }^{7}$ Weikai Xiang, ${ }^{8}$ \\ Huihuang Fang, ${ }^{1}$ Jiaying Mo, ${ }^{1}$ lan Wilkinson, ${ }^{9}$ Yun-Liang Soo, ${ }^{4,5}$ Georg Held, ${ }^{6}$ Kazu Suenaga, ${ }^{3}$ Tong Li, ${ }^{8} \mathrm{Hsin}-\mathrm{Yi}$ \\ Tiffany Chen ${ }^{2, *}$ and Shik Chi Edman Tsang ${ }^{1 *}$ \\ ${ }^{1}$ The Wolfson Catalysis Centre, Department of Chemistry, University of Oxford, Oxford, OX1 3QR, UK \\ ${ }^{2}$ Department of Engineering and System Science, National Tsing Hua University, Hsinchu 300044, Taiwan \\ ${ }^{3}$ National Institute of Advanced Industrial Science and Technology (AIST), Central 5, 1-1-1 Higashi, \\ Tsukuba 305-8565, Japan \\ ${ }^{4}$ National Synchrotron Radiation Research Center, Hsinchu 300, Taiwan \\ ${ }^{5}$ Department of Physics, National Tsing Hua University, Hsinchu 300044, Taiwan \\ ${ }^{6}$ University of Reading, Whiteknights, Reading, Berkshire, RG6 6AH, UK \\ ${ }^{7}$ City University of Hong Kong, Hong Kong \\ ${ }^{8}$ Institute for Materials, Ruhr-Universität Bochum, 44803, Bochum, Germany \\ ${ }^{9}$ Siemens plc, CT NTF, Wharf Road, Oxford OX29 4BP, UK
}

\section{Supporting information}




\section{Supplementary methods}

\section{Experimental procedures}

\section{Chemicals and Materials}

$\mathrm{Mg}\left(\mathrm{NO}_{3}\right)_{2}, \mathrm{MgCl}_{2} \bullet 6 \mathrm{H}_{2} \mathrm{O}$ (reagent grade), benzoic acid, sodium hydroxide (reagent grade), $\mathrm{Ru}_{3}(\mathrm{CO})_{12}$, THF (anhydrous, >99.9\%) were from Sigma-Aldrich. High purity hydrogen gas (99.99\%), nitrogen gas (99.99\%), argon gas (99.99\%), dilute oxygen gas $\left(2.5 \% \mathrm{O}_{2}\right.$ in $\left.\mathrm{He}\right)$, dilute hydrogen gas $\left(5 \% \mathrm{H}_{2}\right.$ in $\left.\mathrm{N}_{2}\right)$ were obtained from BOC. Purity of hydrogen and nitrogen was further enhanced with oxygen, carbon dioxide and moisture traps (HP and $5 \AA$ ) attaching to the gas cylinders.

\section{Materials Synthesis}

\section{MgO preparation}

$\mathrm{MgO}(110)$ was prepared by the calcination under vacuum method. Typically, commercial $\mathrm{MgO}$ (500 mg) was boiled in deionised water for 5 hours. The raw product was then collected by centrifugation and was subsequently dried at $120{ }^{\circ} \mathrm{C}$ for 12 hours. The product was calcined under vacuum at $500{ }^{\circ} \mathrm{C}$ for 6 hours ${ }^{1,2} . \mathrm{MgO}(111)$ was synthesized using the hydrothermal method with the aid of benzoic acid as surfactant. Typically, $\mathrm{MgCl}_{2} \bullet 6 \mathrm{H}_{2} \mathrm{O}(2 \mathrm{~g})$ and benzoic acid $(0.12 \mathrm{~g})$ was dissolved in $60 \mathrm{~mL}$ deionised water. The mixture was stirred for 1 hour. $2 \mathrm{M}$ $\mathrm{NaOH}(20 \mathrm{~mL})$ was then added drop wise into the solution, forming a white precipitate. The slurry was subsequently transferred to a $100 \mathrm{~mL}$ autoclave and gradually heated to $180{ }^{\circ} \mathrm{C}$ and maintained at this temperature for 24 hours. The $\mathrm{Mg}(\mathrm{OH})_{2}$ precursor was obtained after filtration followed by washing with water and drying at $80{ }^{\circ} \mathrm{C}$ under vacuum overnight. Refined synthesis of $\mathrm{MgO}(111)$ nanosheets of optimal surface areas were obtained after calcination in compressed air at $500{ }^{\circ} \mathrm{C}$ for 6 hours ${ }^{1,3-8}$.

\section{Preparation of $\mathrm{Ru} / \mathrm{Mg}(111)$ and $\mathrm{Ru} / \mathrm{MgO}(110)$}

Typically, $\mathrm{Ru}_{3}(\mathrm{CO})_{12}$ was dispersed in THF for 2 hours under sonication. The mixture was then transferred to the $\mathrm{MgO}$ or activated carbon (AC) and allowed to stir at ambient temperature for 8 hours where the solvent is subsequently removed by rotary evaporation. The obtained powder was dried in a $70{ }^{\circ} \mathrm{C}$ oven for 12 hours under vacuum. After that the powder is transferred to a temperature-programmed furnace and heated to $350{ }^{\circ} \mathrm{C}$ for 6 hours under Ar stream to remove the $\mathrm{CO}$ ligand from the $\mathrm{Ru}$ precursor. Loading of $\mathrm{Ru}$ on $\mathrm{MgO}(111)$ and $\mathrm{MgO}(110)$ was confirmed to be 3.4 weight $\%$ and 3.7 weight $\%$ using ICP-MS. 


\section{Characterisation techniques}

\section{Computational details.}

The spin-polarised DFT calculations were performed using the Vienna ab initio Simulation Package (VASP $)^{9-11}$ software. The generalized gradient approximation (GGA) based PerdewBurke-Erzerhof (PBE) functional ${ }^{12}$ was used to include the exchange and correlation effect of the valence electrons. The kinetic energy cutoff of $450 \mathrm{eV}$ was used for all calculations. The project-augmented wave (PAW) ${ }^{13,14}$ method was used to describe the core-valence electron interactions. Each atoms was relaxed until the Hellman-Feynman force criterion were less than $0.01 \mathrm{eV} / \AA$ by using the conjugate gradient minimization algorithm. The bulk structure was optimized using a $10 \times 10 \times 10 \mathrm{Gamma}$ centered grid of $k$-points mesh. The calculated lattice parameters of the bulk MgO is $4.249 \AA$, which are very close to the corresponding experimental values of $4.21 \AA$ (calculated from XRD using Bragg's Law).

The pure $\mathrm{MgO}(111)$ surface was modeled using a twelve-layer slab repeated in a $2 \times 2$ surface unit cell; Symmetric slab model was considered for optimization by fixing the middle four layers to balance the dipole-moment. The $k$-point mesh was sampled using a $1 \times 1 \times 1 \mathrm{Gamma}$ centered grid. To avoid interactions between slabs, all slabs were separated by a vacuum gap greater than $15 \AA$. After complete convergence testing studies, the geometric and electronic structures of the slabs are analyzed. The charge transfer was investigated with Bader charge analysis. We used a Gaussian smearing with $\sigma=0.01 \mathrm{eV}$ for Density of state analysis.

The $\mathrm{H}$ adsorption energy $\left(E_{\mathrm{ads}}\right)$ on the clean substrate was calculated by:

$E_{\mathrm{ads}}=E(\mathrm{H} / \mathrm{sub})-E(\mathrm{sub})-1 / 2 E(\mathrm{H} 2)$,

where $E\left({ }_{\mathrm{H} / \mathrm{sub}}\right), E(\mathrm{sub})$, and $E\left(\mathrm{H}_{2}\right)$ are the DFT energies of the adsorption complex, clean substrate, and gas-phase $\mathrm{H}_{2}$ molecule, respectively.

The $\mathrm{H}$ adsorption energy on $\mathrm{Ru} / \mathrm{MgO}$ was calculated by:

$E_{a d s}=\left\{E_{(n H R u / M g O)}-E_{(R u / M g O)}-\mathrm{n} / 2 E_{(H 2)}\right\} / \mathrm{n}$

where $\left.E_{(n H R u / M g O)}, E_{(R u / M g O}\right)$, and $E\left(\mathrm{H}_{2}\right)$ are the DFT energies of the adsorption complex, main catalyst, and gas-phase $\mathrm{H}_{2}$ molecule, respectively while $\mathrm{n}$ is number of $\mathrm{H}$ atoms.

The binding energy of $\mathrm{Ru}$ atom on $\mathrm{MgO}$ surfaces was calculated by:

$E_{b}=\mathrm{E}_{(R u / M g O)}-\mathrm{E}_{(R u)}-\mathrm{E}_{(M g O)}$ (w.r.t. Ru atom)

where $E_{(R u / M g O)}, E_{(M g O)}$, and $\mathrm{E}_{(R u)}$ are the DFT energies of the adsorption complex, clean substrate, and $\mathrm{Ru}$ atom in gas-phase, respectively. 


\section{In-situ XPS measurements}

The study of the Ru charge distribution and the corresponding change in $[\mathrm{OH}]$ concentration under different gas conditions was performed by means of operando XPS measurements, at the Diamond Light Source B07 beamline. The powdered samples were dissolved in acetone and mounted on a silicon wafer, which were subsequently heated by a heat plate at $110^{\circ} \mathrm{C}$ to remove the solvent. The temperature was maintained at a constant throughout the experiment at 350 ${ }^{\circ} \mathrm{C}$. The measurements were performed either under 1 mbar $\mathrm{Ar}$ or $1 \mathrm{mbar}^{\mathrm{H}} \mathrm{H}_{2}$. The gas composition was continuously monitored by online mass spectrometry (MS). If not otherwise stated, $\mathrm{Ru} 3 \mathrm{~d}, \mathrm{O}$ 1s, and $\mathrm{Mg} 2 \mathrm{~s}$ spectra were recorded using a fixed photon energy of $950 \mathrm{eV}$. The binding energy (BE) scale was calibrated with respect to the $\mathrm{Mg} 2 \mathrm{~s}(88.1 \mathrm{eV})$ as internal standard.

\section{In-situ FTIR measurements}

The Fourier-Transform Infra-Red (FTIR) experiments were carried out using a Thermo Scientific Nicolet 6700 spectrometer equipped with CaF2 windows at $200{ }^{\circ} \mathrm{C}$. Spectra were obtained by collecting 32 scans with a resolution of $4 \mathrm{~cm}^{-1}$ and are presented in absorbance unit. Powder of freshly prepared samples were pressed into pellets and loaded onto the sample holder. The sample was then flushed with He for 30 minutes to clean the surface under $250{ }^{\circ} \mathrm{C}$ followed by ramping down to $200{ }^{\circ} \mathrm{C}$ for measurement. After collecting background spectra, $\mathrm{N}_{2}$ gas was passed through the sample holder for 15 minutes at $20 \mathrm{~mL} \mathrm{~min}^{-1}$ and in-situ sample spectra were recorded. After 15 minutes, $\mathrm{H}_{2}$ gas was then passed at $20 \mathrm{~mL} \mathrm{~min}^{-1}$ and in-situ spectra were recorded. The presented spectra are the difference spectra between the in-situ spectra and the background spectra.

\section{APT measurements}

APT relies on generating high electric fields $\left(\sim 3-5 \mathrm{~V} \AA^{-1}\right)$ at the apex of a needle-shaped specimen. On top of this, an additional short (ns) voltage or laser pulse was applied to remove ions in a controlled manner into a high-resolution time-of-flight mass spectrometer equipped with a single-ion-sensitive detector. APT thus can provide 3D elemental mapping with singleatom sensitivity.

To prepare the APT specimens, nanoparticles were first distributed on a Ni-coated Si flat wafer and subsequently embedded by a 150-nm thick protective Ni layer in Leica EM ACE600 high vacuum sputter coater ${ }^{4}$. The needle-shaped APT specimens were then prepared by a standard lift-out procedure using a focused ion beam/scanning electron microscope (FIB/SEM) in FEI G4 CX. The APT experiments were conducted in a CAMECA LEAP 5000 XR instrument at a specimen temperature of $50 \mathrm{~K}$, a detection rate of 0.5 , a pulsing rate of $125 \mathrm{kHz}$, and a laser pulse energy of $30 \mathrm{pJ}$. Data reconstruction and analysis were performed using the commercial IVAS 3.8.2 ${ }^{\mathrm{TM}}$ software.

\section{${ }^{1} \mathrm{H}$ NMR measurements}

Static ${ }^{1} \mathrm{H}$ MAS NMR spectra were taken to investigate the hydrogenic species on $\mathrm{MgO}(111)$ and $\mathrm{Ru}-\mathrm{MgO}(111)$, respectively after their pre-reduction in $\mathrm{H}_{2}$. Before data collection, samples 
were pretreated using the following procedure. They were first placed in glass tubes connected to a vacuum line or $\mathrm{H}_{2}$ gas at $350{ }^{\circ} \mathrm{C}$ for $1 \mathrm{~h}$. After pre-reduction in flowing $\mathrm{H}_{2}$ in 1 atmosphere, the samples were cooled to room temperature, Ar gas was introduced to the samples. Sample tubes were sequentially flame-sealed and transferred into a $\mathrm{ZrO}_{2}$ rotor with a kel-F end-cap in a glovebox. NMR measurements were carried out at $298 \mathrm{~K}$ using a Bruker Avance III $400 \mathrm{MHz}$ spectrometer at the Larmor frequencies of 399.33 for ${ }^{1} \mathrm{H}$.

\section{Synchrotron X-ray absorption fine structure (XAFS)}

XAFS spectra for the $\mathrm{Ru} / \mathrm{MgO}(111)$ sample was recorded at the $\mathrm{Ru} \mathrm{K}$ absorption edge, in fluorescence mode using a Lytle fluorescence detector, under reaction conditions, at beamline BL07A of the Taiwan light source at National Synchrotron Radiation Research Center in Taiwan. A Si (111) Double Crystal Monochromator( DCM) was used to scan the photon energy. The energy resolution for the incident X-ray photons was estimated to be $2 \times 10^{-4}$. The Demeter software package (Athena and Artemis) was used for XAFS data analysis for the Ru data. To ascertain the reproducibility of the experimental data, at least two scan sets were collected and compared for each ex-situ sample. The spectra were calibrated with foils as a reference. And the amplitude parameter was obtained from EXAFS data analysis of the Ru foil, which was used as a fixed input parameter in the data fitting to allow the refinement in the coordination number of the absorption element. In this work, the first shell data analyses under the assumption of single scattering were performed with the errors estimated by R-factor.

For the in-situ measurements the spectrum was obtained in fluorescence mode using a fixed bed reactor with Kapton windows to allow synchrotron X-rays to pass through. The temperature was controlled with a Eurotherm controller with a thermocouple positioned in the centre of the heating block. For safety reasons, dilute gas mixtures were used. $\mathrm{H}_{2} / \mathrm{He}\left(5 \% \mathrm{H}_{2}\right.$ balanced in $\mathrm{He}, \mathrm{BOC}$ ) and pure He gases were introduced to the heated chamber containing the fixed bed of catalysts with flow rates controlled by mass flow controllers. The reactor was heated to $300{ }^{\circ} \mathrm{C}$ at a ramp rate of $5{ }^{\circ} \mathrm{C} / \mathrm{min}$ and held at temperature for $30 \mathrm{~min}$ under a flow of $\mathrm{H}_{2} / \mathrm{He}$ for pre-reduction. Then it was switched to $\mathrm{He}$ and flowed for 30 minutes. XAFS spectrum was then obtained until there is no change in the absorption edge. 


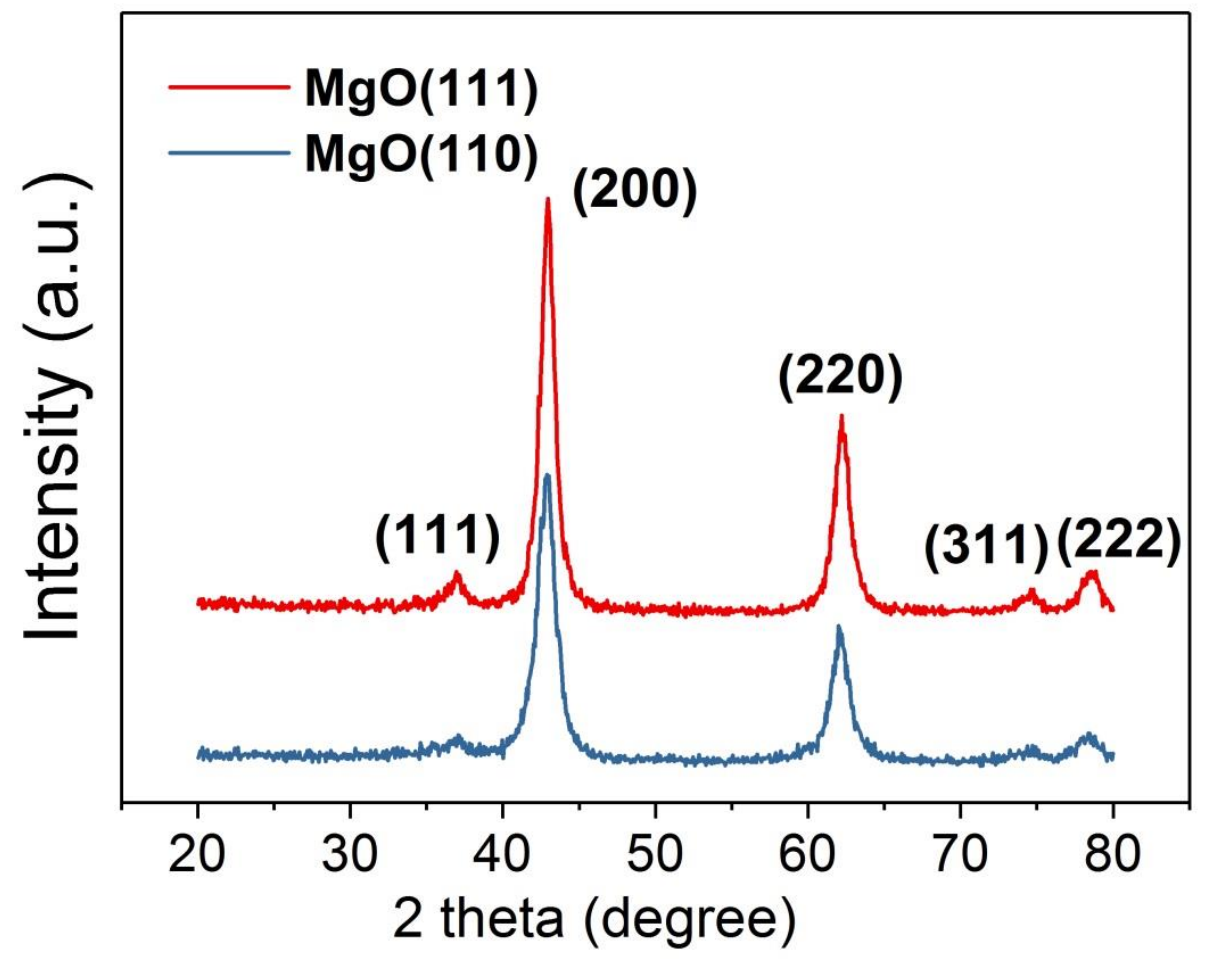

Figure S1. Phase identification of $\mathrm{MgO}(111)$ and $\mathrm{MgO}(110)$ confirmed by X-ray diffraction. 


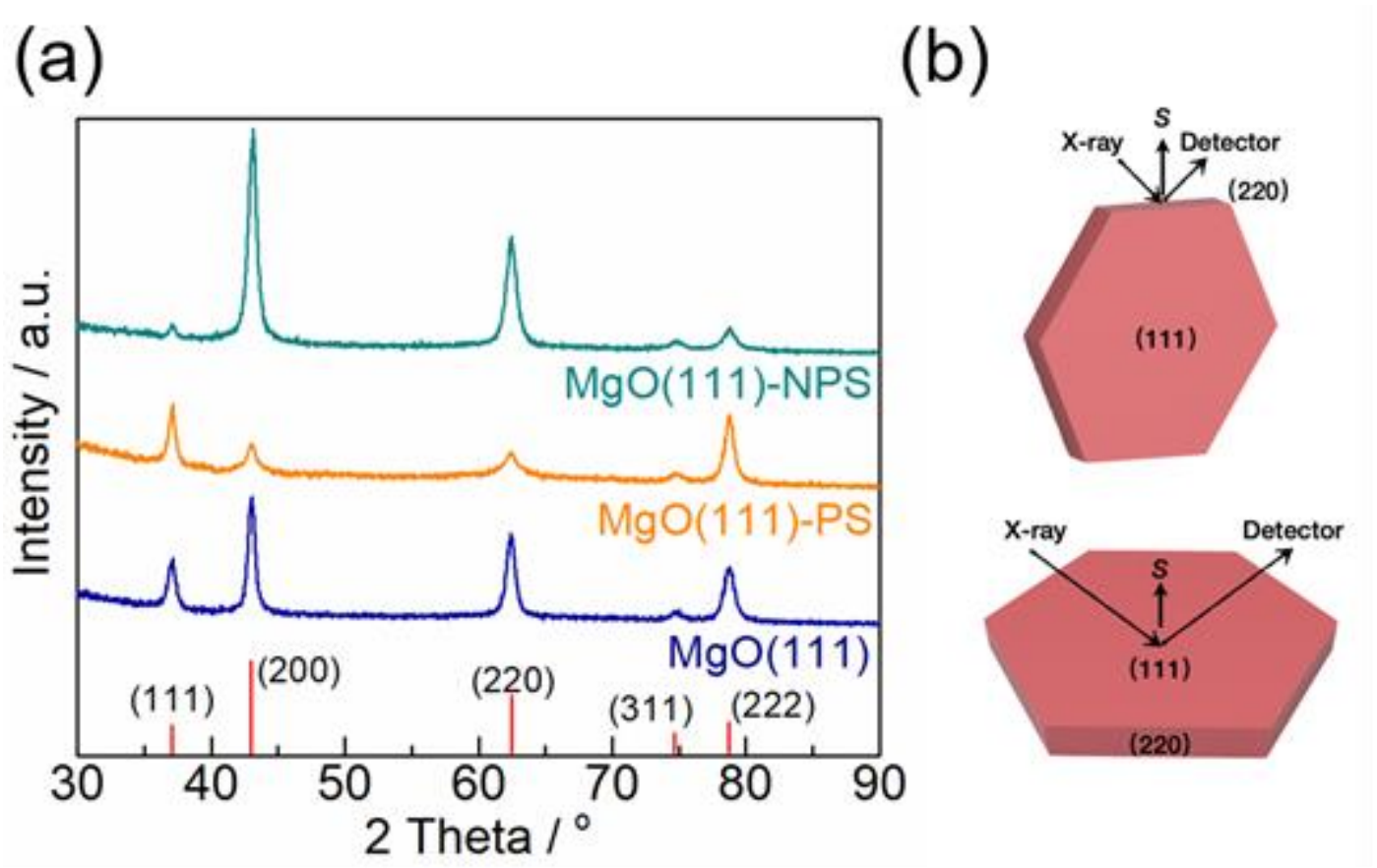

Figure S2 (a) XRD patterns of $\mathrm{MgO}(111)$ with no dispersion [MgO(111)], ethanol-dispersion [MgO(111)-PS], and n-hexane-dispersion [MgO(111)-NPS]. PS stands for polar solvent (ethanol) whereas NPS stands for non-polar solvent (hexane); (b) schematic illustration of MgO nanosheets where X-ray beams incident at different exposed facet. Top panel: diffraction vector is parallel to the (111) facet; Bottom panel: diffraction vector is perpendicular to the (111) facet. 

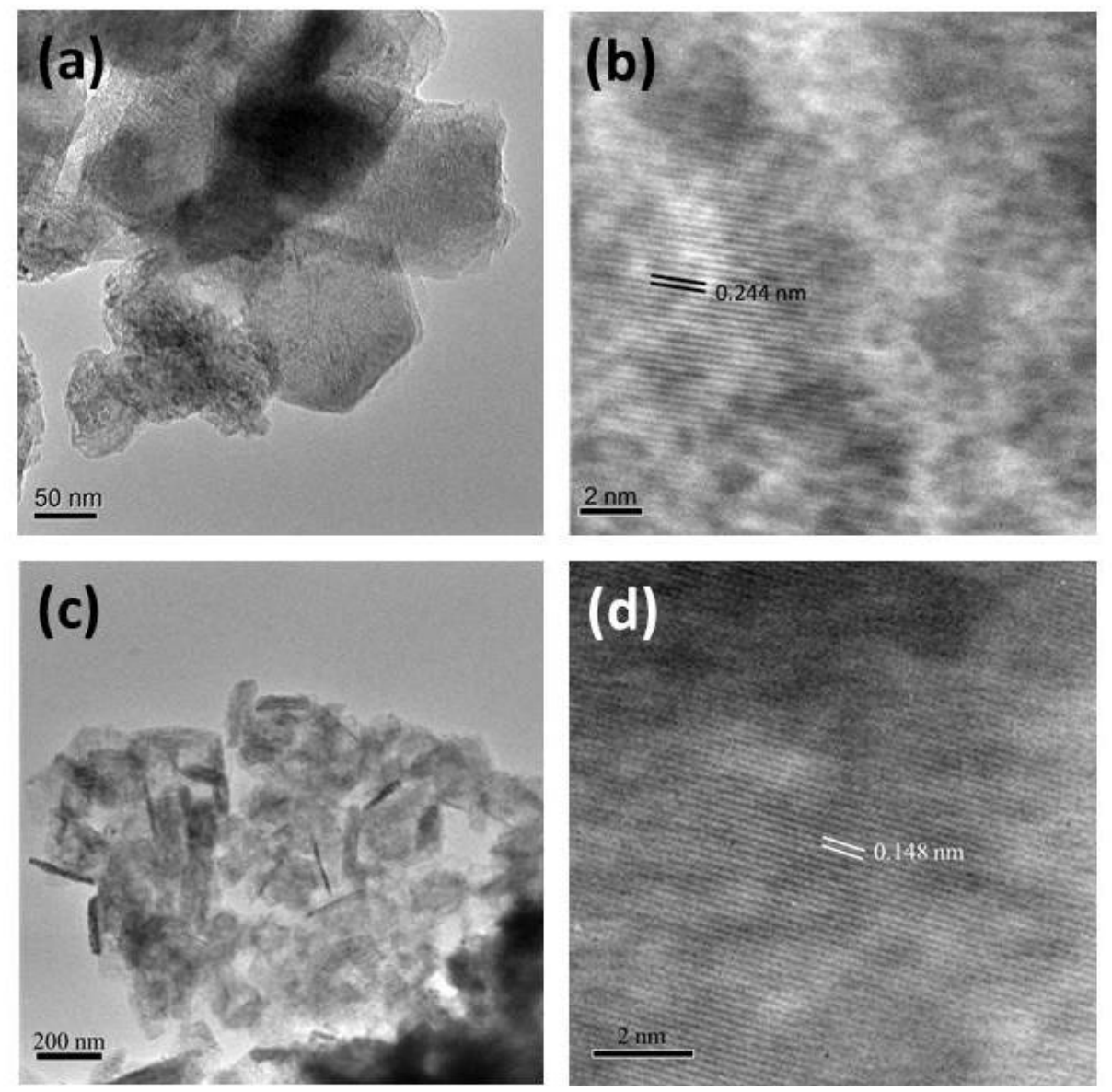

Figure S3. TEM and HRTEM images of (a-b) $\mathrm{MgO}(111)$, (c-d) $\mathrm{MgO}(110)$. Lattice spacing on each exposed plane is calculated from the average of 10 lattice spacing. Both of the values for $\mathrm{MgO}(111)$ and $\mathrm{MgO}(110)$ matches well with the literature ${ }^{7,8}$. 
(a)

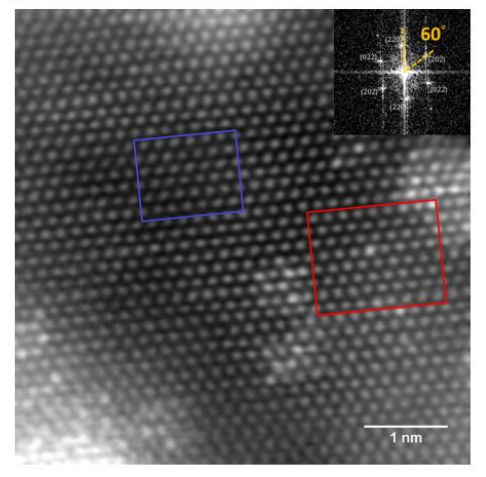

(c)

(b)

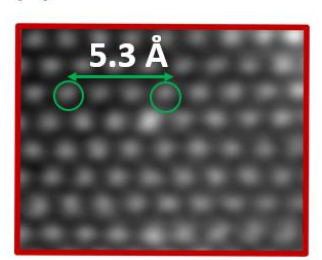

(d)
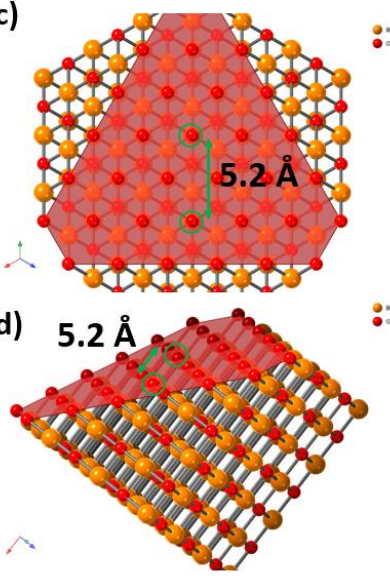

View from [111]

top

View from side

Figure S4. (a) HAADF-STEM image of $3.4 \mathrm{wt} \%-\mathrm{Ru} / \mathrm{MgO}(111)$ observed from [111] direction. The bright spots are confirmed to be $\mathrm{Ru}$ from EELS; individual $\mathrm{Ru}$ on trigonal OOO hollow sites and 2-D islands with no $\mathrm{Ru}-\mathrm{Ru}$ lattice formed probably via surface Ostwald ripening can be seen. The inset in (a) displays the corresponding FFT pattern of the pure faceted $\mathrm{MgO}(111)$ region in the blue rectangle; (b) Enlarged pattern of the region in the red rectangle in (a). The distance between 4 oxygen atoms is measured to be $5.3 \pm 0.2 \AA$; (c) Simulated model of $\mathrm{MgO}(111)$ with exposed oxygen-terminated surface (Red: $\mathrm{O}$, Orange: $\mathrm{Mg}$ ). The view direction is from [111]. The [111] lattice plane (red) is displayed for reference. The distance between 4 atoms ( 2 atoms in the [111] plane and 2 atoms are from layers below) is $5.2 \AA$ : thus, the pattern and the distance match well with the measured value in the experimental STEM image; (d) The side view of the $\mathrm{MgO}(111)$ model in (c). 

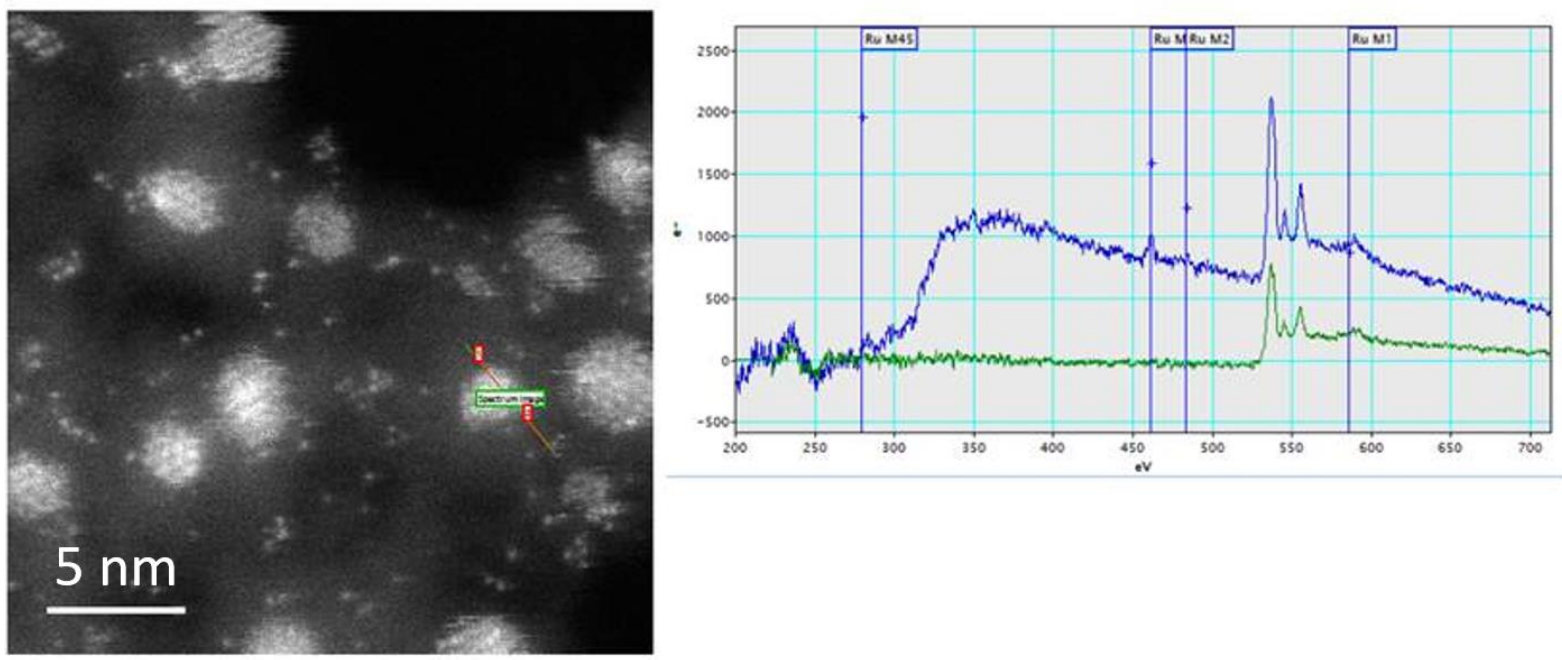

Figure S5. STEM image of $\mathrm{Ru} / \mathrm{MgO}(110)$. The presence of $\mathrm{Ru}$ is identified by EELS mapping. All the $\mathrm{Ru}$ species are in the form of nanoparticles (nps) with matched Ru-Ru metal lattice. The tiny white dots shown in the image might possibly be due to contamination since EELS displays no signal for the Ru M edges in contrast to the nanoparticles. The exposed plane where $\mathrm{Ru}$ nps were sitting on was however not clearly defined, which might be caused by significant charging effect due to the insulator nature. 


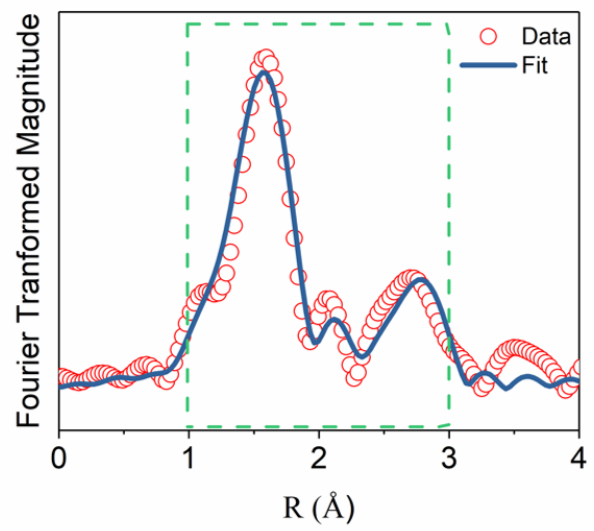

\begin{tabular}{|l|l|l|l|}
$\begin{array}{l}\text { Scattering } \\
\text { Path }\end{array}$ & $\begin{array}{l}\text { Bond Length } \\
(\AA)\end{array}$ & $\begin{array}{l}\text { Coordination } \\
\text { No. }\end{array}$ & $\begin{array}{l}\text { Debye Waller's } \\
\text { factor }\end{array}$ \\
\hline Ru-O & $2.03(2)$ & $3.0(2)$ & 0.009 \\
\hline Ru-Mg & $3.17(3)$ & $1.4(3)$ & 0.003
\end{tabular}

Figure S6. Fourier Transformed of Ru K-edge EXAFS spectra for $\mathrm{Ru} / \mathrm{MgO}(111)$ measured at $300{ }^{\circ} \mathrm{C}$ under flowing He after hydrogen pre-reduction. Parameters obtained from least-square fitting are shown on the right. Parameters used: k-range: 3-12, R-range: 1-3, R-factor: $1.9 \%$. 


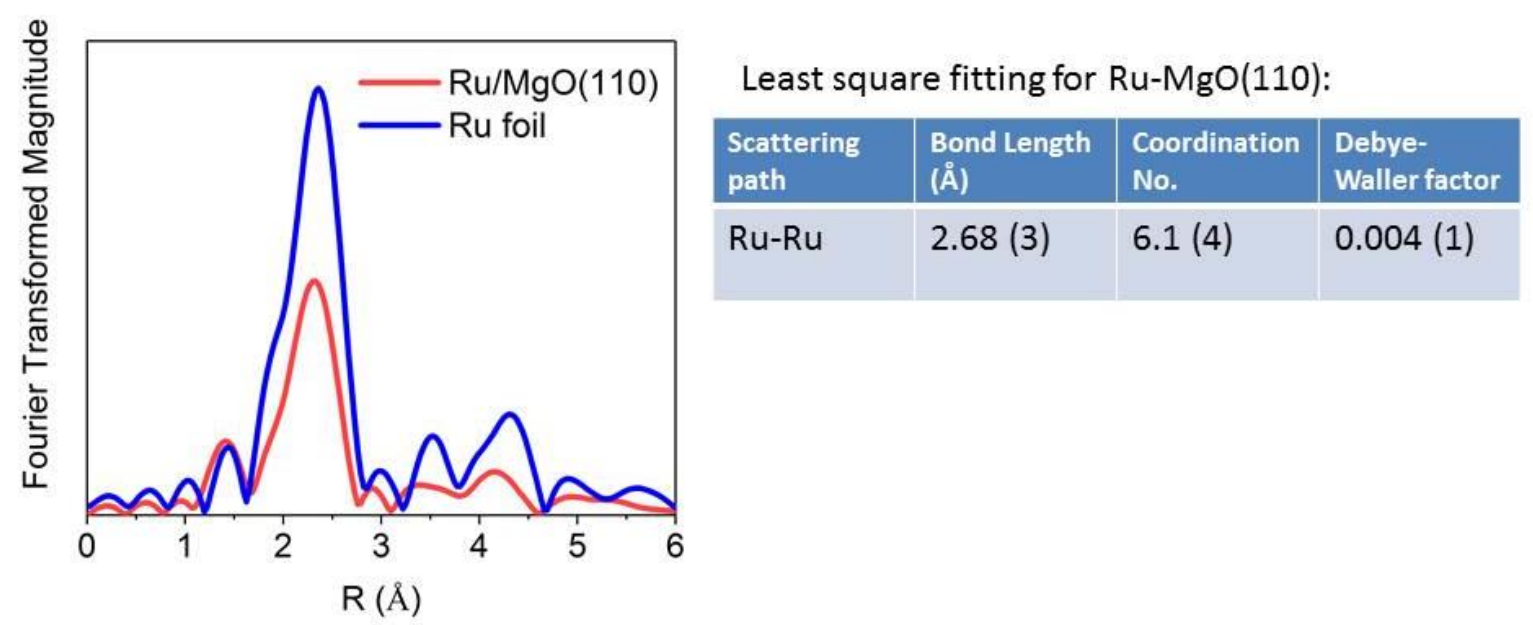

Figure S7. Fourier Transformed of Ru K-edge EXAFS spectra for $\mathrm{Ru} / \mathrm{MgO}(110)$ and $\mathrm{Ru}(0)$ foil at ambient temperature. Parameters obtained from least-square fitting are shown on the right. Parameters used: k-range: 3-11.4, R-range: 1.35-3, R-factor: 2.0\%. 


Initial
Structure

Figure S8. Density Functional Theory calculations give the optimised geometry of a single Ru atom on hollow $\mathrm{OOO}$ atop to $\mathrm{Mg}$ as the most stable surface site of $\mathrm{MgO}(111)$. 


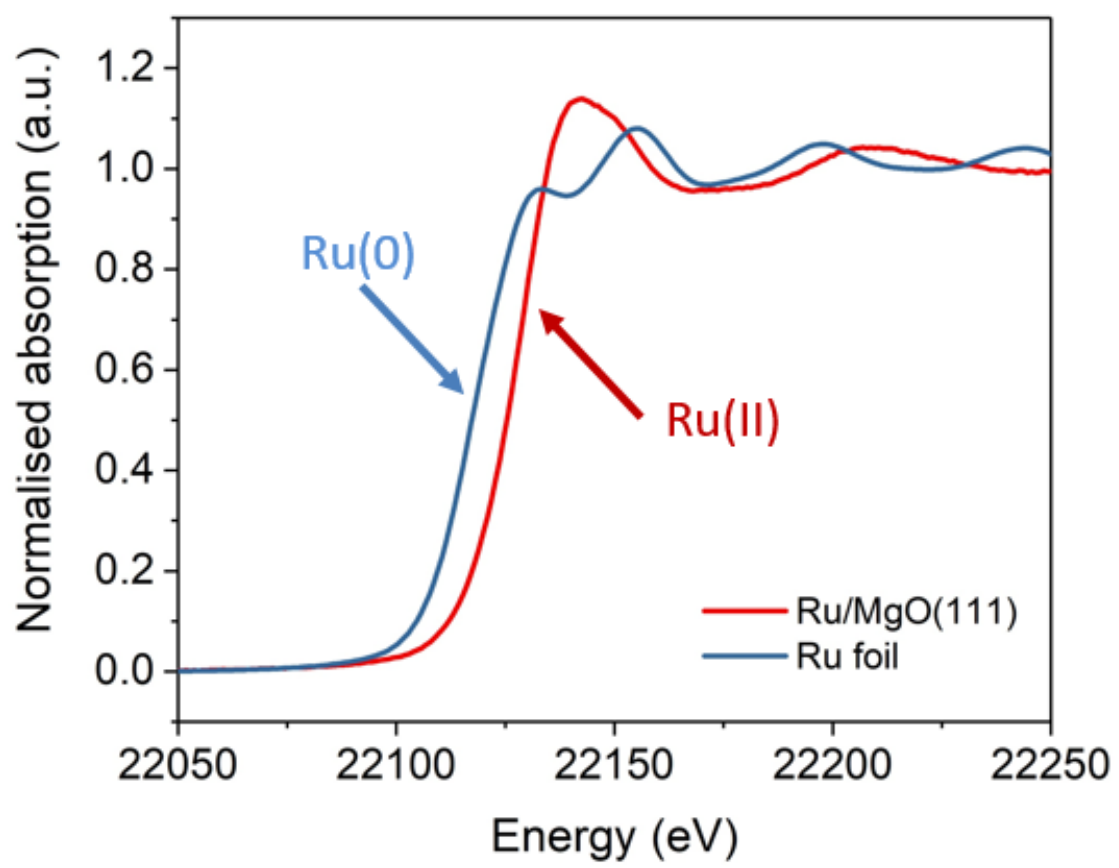

Figure S9. In-situ XANES measured at $300{ }^{\circ} \mathrm{C}$ under flowing He. The shift towards higher binding energy indicates a positive oxidation state of $\mathrm{Ru}$. 
(a)

\begin{tabular}{|c|c|c|c|c|c|c|}
\hline \multicolumn{3}{|c|}{ System } & $\mathrm{MgO}(111)$ & $\mathrm{Ru} / \mathrm{MgO}(111)$ & $\mathrm{MgO}(110)$ & $\mathrm{Ru} / \mathrm{MgO}(110)$ \\
\hline \multicolumn{3}{|c|}{ Adsorption Site } & - & $\begin{array}{l}\text { O-O-O Hollow } \\
\text { atop Mg }\end{array}$ & - & $\begin{array}{l}\text { O-O Bridge } \\
\text { atop } \mathrm{Mg}\end{array}$ \\
\hline \multicolumn{3}{|c|}{$\mathbf{E}_{\text {bind }}(\mathrm{eV})$} & - & -10.81 & - & -3.18 \\
\hline \multirow[t]{11}{*}{$\mathbf{q}|\mathbf{e}|$} & \multicolumn{2}{|c|}{$\mathbf{R u}$} & - & +1.270 & - & -0.238 \\
\hline & \multirow[t]{3}{*}{$\begin{array}{l}\text { Over } \\
\text { layer }\end{array}$} & $\begin{array}{c}O \text { bind } \\
\text { with } R u\end{array}$ & - & -1.050 & - & -1.447 \\
\hline & & $\begin{array}{c}\text { O no } \\
\text { bind with } \\
\text { Ru }\end{array}$ & -0.971 & -1.504 & -1.576 & - \\
\hline & & Mg & +1.633 & +1.637 & +1.603 & +1.574 \\
\hline & \multicolumn{2}{|c|}{$O$ unfixed in bulk } & -1.643 & -1.640 & -1.634 & -1.636 \\
\hline & \multicolumn{2}{|c|}{ O fixed in bulk } & -1.655 & -1.655 & -1.631 & -1.631 \\
\hline & \multicolumn{2}{|c|}{$\begin{array}{c}\text { Mg unfixed in } \\
\text { bulk }\end{array}$} & +1.640 & +1.641 & +1.628 & +1.632 \\
\hline & \multicolumn{2}{|c|}{ Mg fixed in bulk } & $+1.655 /$ & +1.655 & +1.631 & +1.631 \\
\hline & \multirow{2}{*}{$\begin{array}{c}\text { Botto } \\
\text { m } \\
\text { layer }\end{array}$} & Mg & +1.076 & +1.064 & +1.603 & +1.575 \\
\hline & & $\mathbf{O}$ & -1.830 & $\begin{array}{c}-1.860 / \\
-\end{array}$ & $\begin{array}{c}-1.583 / \\
-\end{array}$ & -1.583 \\
\hline & \multicolumn{2}{|c|}{ Total MgO } & 0.000 & -1.270 & 0.000 & +0.238 \\
\hline
\end{tabular}

(b)

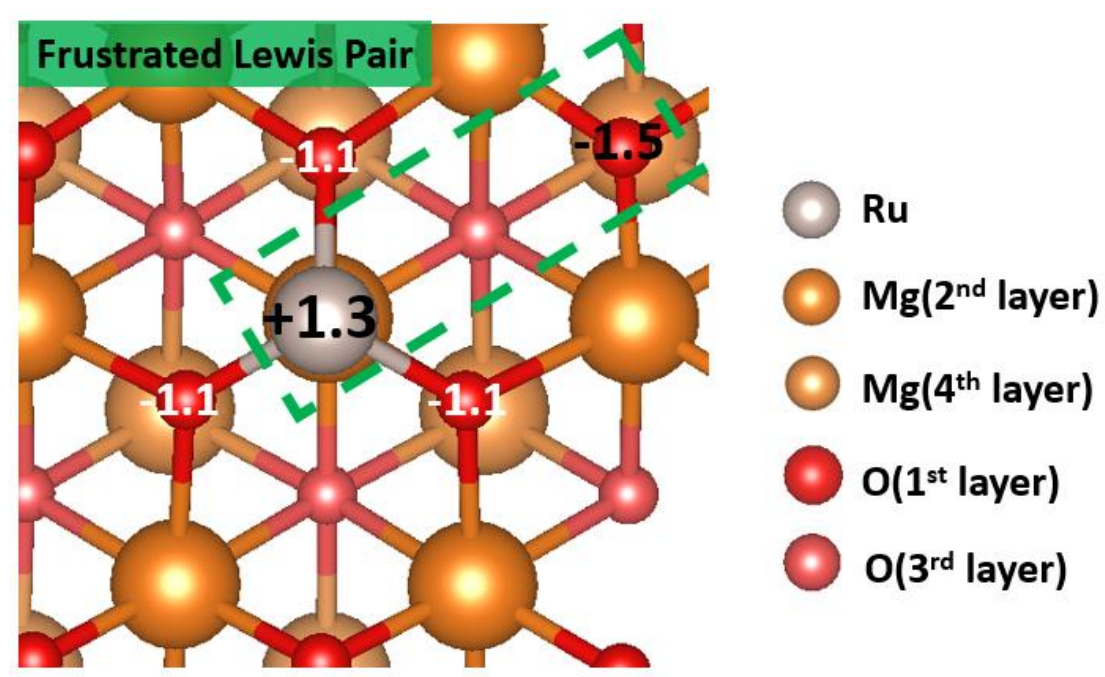

Figure S10. (a) Bader charge analysis of surface Ru and O on the $\mathrm{Ru} / \mathrm{MgO}(111)$; (b) Optimised structure of $\mathrm{Ru} / \mathrm{MgO}(111)$ depicting the charge on the surface atoms. 


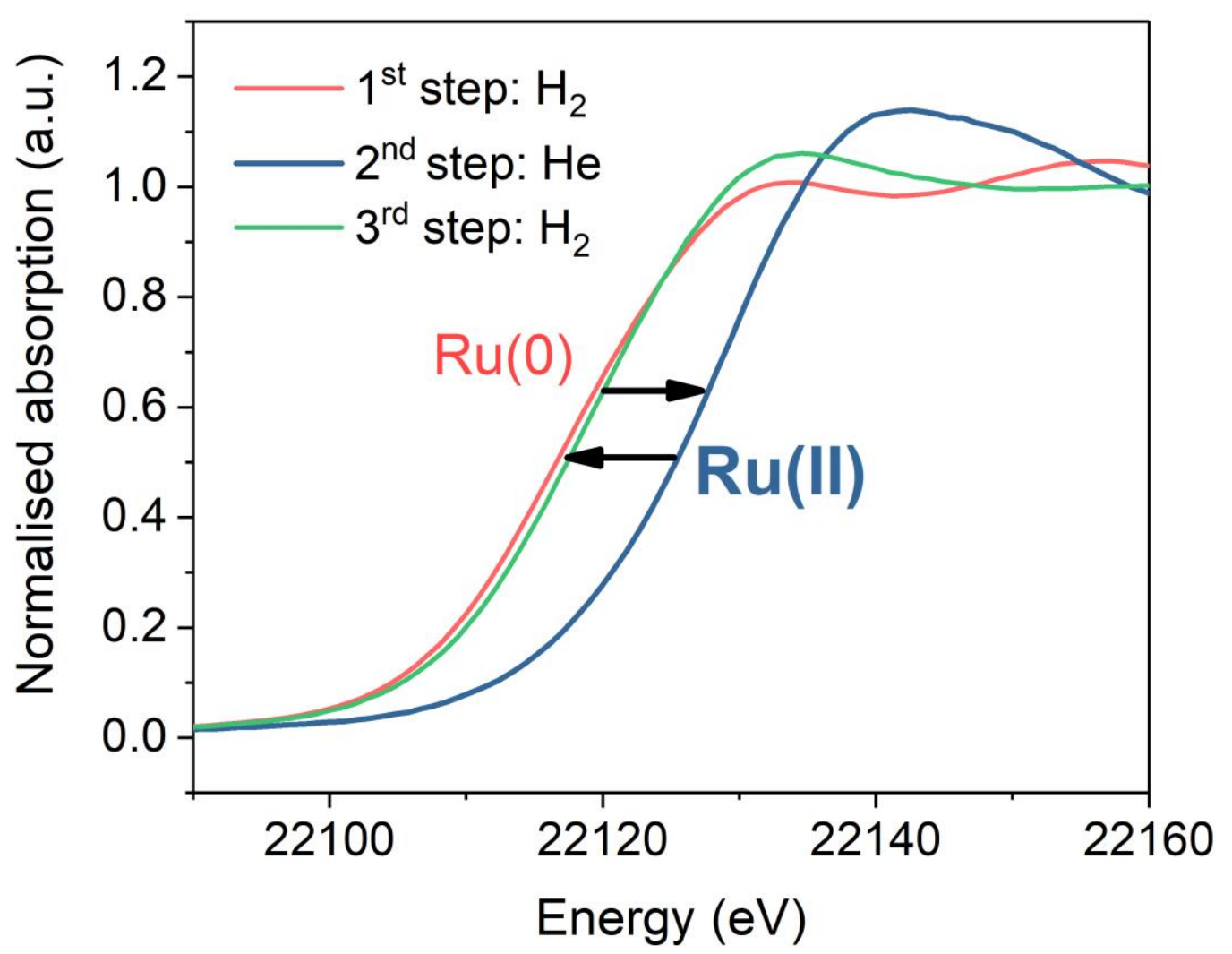

Figure S11. In-situ XANES for $\mathrm{Ru} / \mathrm{MgO}(111)$ under dynamic switching between $5 \% \mathrm{H}_{2} / \mathrm{He}$ and $\mathrm{He}$ at $300{ }^{\circ} \mathrm{C}$.

The XANES spectra for Ru K-edge (of the Ru-MgO(111) sample) indicates a zero oxidation state when it is under hydrogen, but quickly swings towards higher binding energy upon switching from hydrogen to helium. This suggests an increase in the oxidation state for the Ru when the sample is out of the reducing atmosphere. As a result, a continuous driving force is needed to keep the $\mathrm{Ru}$ at a reduced state with corresponding coverage of $\mathrm{H}^{+}$on the oxide support surface. In our case, under a hydrogen atmosphere, the forward hydrogen spillover process causes the adsorbed hydrogen atom to split into proton that migrate towards the oxygen-terminated surface of the support and electron that reduces the $\mathrm{Ru}^{2+}$ to $\mathrm{Ru}(0)$. When the hydrogen atmosphere is removed, the hydroxyl proton on the support undergoes reversespillover where the $\mathrm{H}^{+}$abstracts the electron from $\mathrm{Ru}$ and becomes $\mathrm{H}$ atom again. This echoes with the AP-XPS experiment showing a reduction in the $[\mathrm{OH}]$ shoulder and corresponding increase in the $\left[\mathrm{O}^{2-}\right]$ component when the gas feed is switched from hydrogen to argon (Figure $3 \mathrm{~b}$ in the maintext). 
(a)

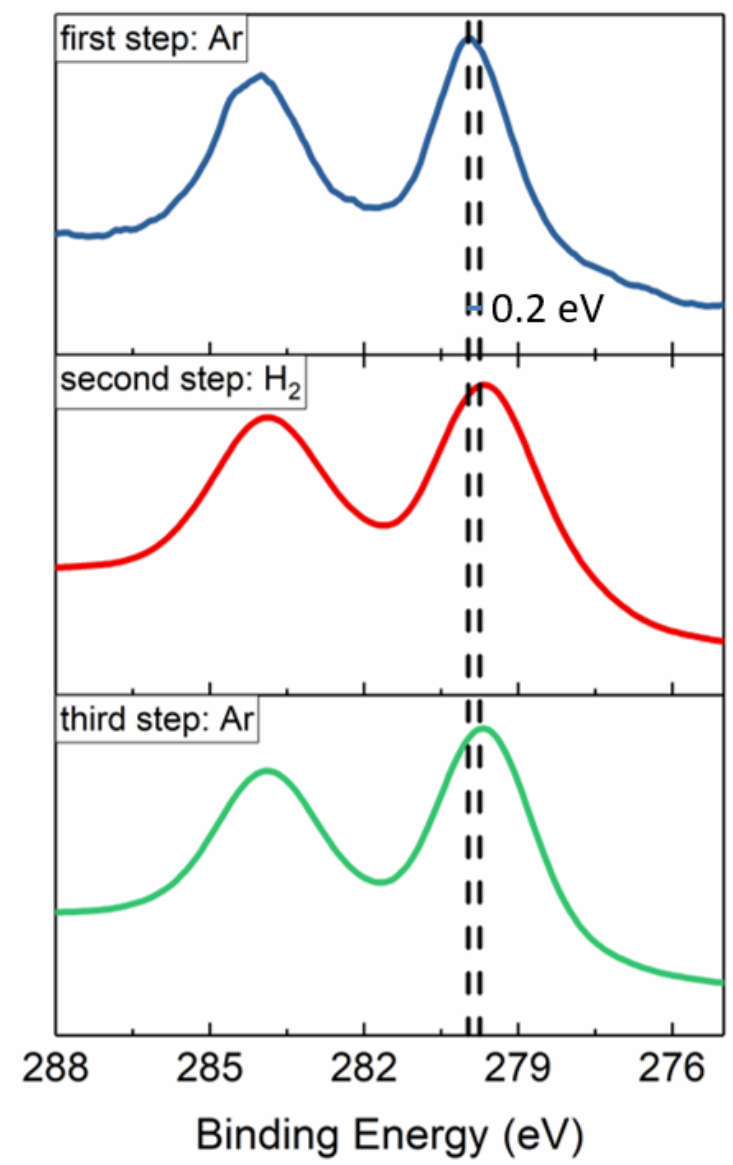

(b)

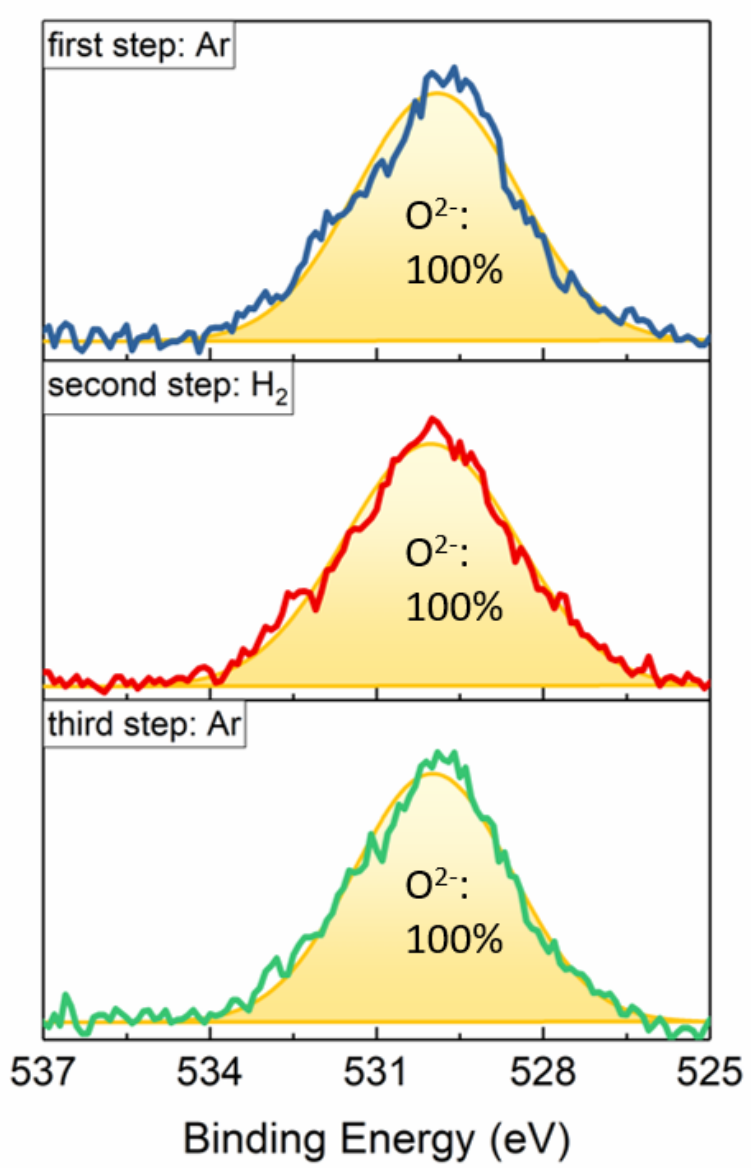

Figure S12. In-situ AP-XPS for $\mathrm{Ru} / \mathrm{MgO}(110)$ under 1 mbar $\mathrm{Ar}$ and $\mathrm{H}_{2}$ at $350{ }^{\circ} \mathrm{C}$ : (a) $\mathrm{Ru} 3 \mathrm{~d}$. (b) O1s spectra. 


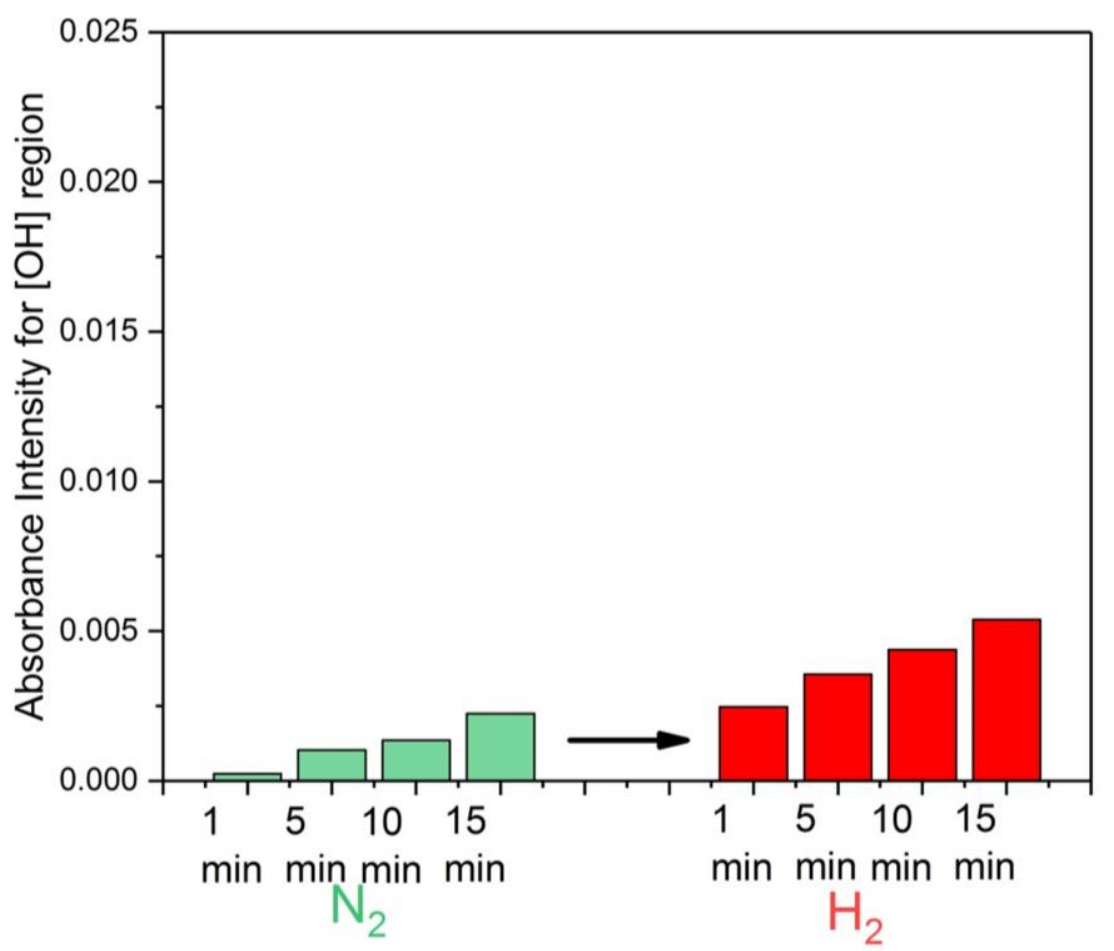

Figure S13. In-situ DRIFT for $\mathrm{Ru} / \mathrm{MgO}(110)$ under 1 bar $\mathrm{N}_{2}$ and $\mathrm{H}_{2}$ at $200{ }^{\circ} \mathrm{C}$. 


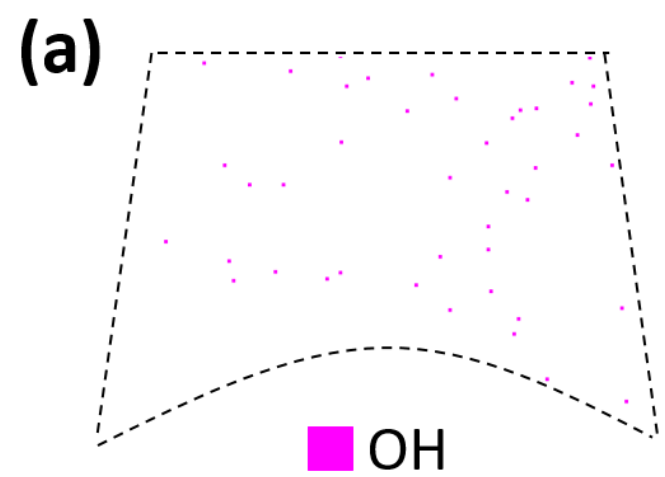

(b)

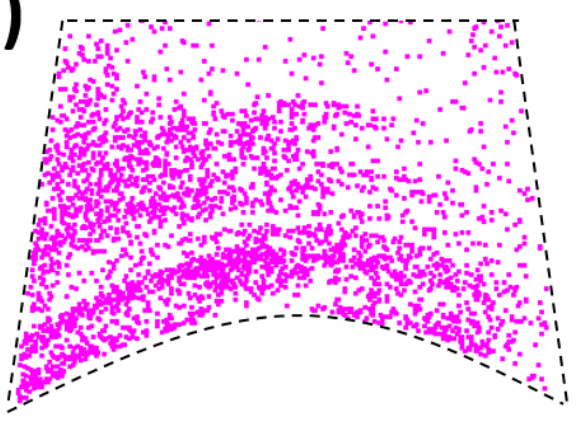

\section{Before Hydrogen Dosage}

\section{After Hydrogen Dosage}

Figure S14. Atomic Probe Tomography of Ru/MgO(111) (a) before hydrogen dosage; (b) after hydrogen dosage. (Pink: $\mathrm{OH}$ )

(a)

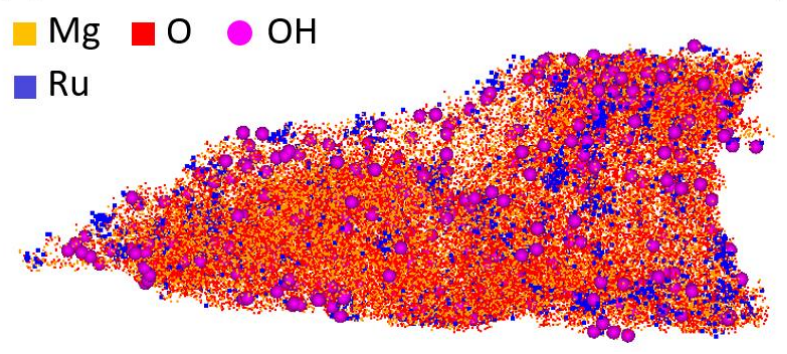

(b)

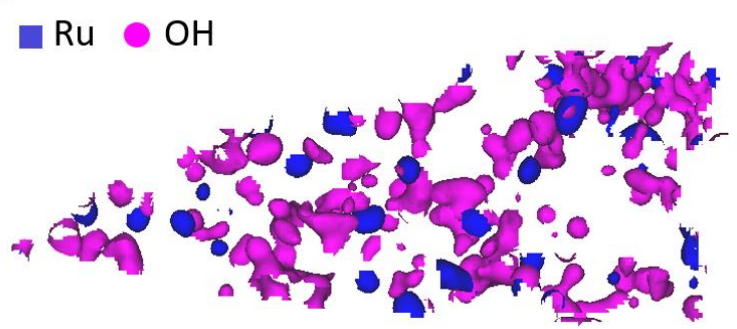

Figure S15. Atomic Probe Tomography Ru/MgO(111) (a) 3D atomic map of Ru, OH distribution on $\mathrm{MgO}$; (b) Isosurface map of $\mathrm{Ru}, \mathrm{OH}$ distribution (Mg: orange; O: red; $\mathrm{Ru}$ : blue; $\mathrm{OH}$ : pink) 


\section{References}

(1) Verziu, M.; Cojocaru, B.; Hu, J.; Richards, R.; Ciuculescu, C.; Filip, P.; Parvulescu, V. I. Sunflower and Rapeseed Oil Transesterification to Biodiesel over Different Nanocrystalline MgO Catalysts. Green Chem. 2008, 10, 373-381.

(2) Richards, R.; Li, W.; Decker, S.; Davidson, C.; Koper, O.; Zaikovski, V.; Volodin, A.; Rieker, T.; Klabunde, K. J. Consolidation of Metal Oxide Nanocrystals. Reactive Pellets with Controllable Pore Structure That Represent a New Family of Porous, Inorganic Materials. J. Am. Chem. Soc 2000, 122, 4921-4925.

(3) Zhu, K.; Hu, J.; Kübel, C.; Richards, R. Efficient Preparation and Catalytic Activity of $\mathrm{MgO}(111)$ Nanosheets. Angew. Chemie Int. Ed. 2006, 45, 7277-7281.

(4) $\mathrm{Hu}$, J.; Zhu, K.; Chen, L.; Kübel, C.; Richards, R. MgO(111) Nanosheets with Unusual Surface Activity. J. Phys. Chem. C 2007, 111, 12038-12044.

(5) Hu, J.; Song, Z.; Chen, L.; Yang, H.; Li, J.; Richards, R. Adsorption Properties of $\mathrm{MgO}(111)$ Nanoplates for the Dye Pollutants from Wastewater. J. Chem. Eng. Data 2010, 55, 3742-3748.

(6) Li, Z.; Ciobanu, C. V.; Hu, J.; Palomares-Báez, J. P.; Rodríguez-López, J. L.; Richards, R. Experimental and DFT Studies of Gold Nanoparticles Supported on $\mathrm{MgO}(111)$ Nano-Sheets and Their Catalytic Activity. Phys. Chem. Chem. Phys. 2011, $13,2582-2589$.

(7) Chen, J.; Tian, S.; Lu, J.; Xiong, Y. Catalytic Performance of MgO with Different Exposed Crystal Facets towards the Ozonation of 4-Chlorophenol. Appl. Catal. A Gen. 2015, 506, 118-125.

(8) Wang, F.; Ta, N.; Shen, W. MgO Nanosheets, Nanodisks, and Nanofibers for the Meerwein-Ponndorf-Verley Reaction. Appl. Catal. A Gen. 2014, 475, 76-81.

(9) Kresse, G.; Furthmüller, J. Efficient Iterative Schemes for Ab Initio Total-Energy Calculations Using a Plane-Wave Basis Set. Phys. Rev. B 1996, 54, 11169-11186.

(10) Kresse, G.; Furthmüller, J. Efficiency of Ab-Initio Total Energy Calculations for Metals and Semiconductors Using a Plane-Wave Basis Set. Comput. Mater. Sci. 1996, $6,15-50$.

(11) Kresse, G.; Hafner, J. Ab Initio Molecular Dynamics for Liquid Metals. Phys. Rev. B 1993, 47, 558-561.

(12) Perdew, J. P.; Burke, K.; Ernzerhof, M. Generalized Gradient Approximation Made Simple. Phys. Rev. Lett. 1996, 77, 3865-3868.

(13) Blöchl, P. E. Projector Augmented-Wave Method. Phys. Rev. B 1994, 50, 1795317979.

(14) Kresse, G.; Joubert, D. From Ultrasoft Pseudopotentials to the Projector AugmentedWave Method. Phys. Rev. B - Condens. Matter Mater. Phys. 1999, 59, 1758-1775. 
